\title{
L'effondrement du tunnel de chemin de fer de Vierzy
}

P. HABIB

Ancien Président du Comité français de mécanique des roches

Conseiller scientifique

du LMS

(École polytechniqueÉcole des mines de Paris)

LMS

École polytechnique 91128 Palaiseau Cedex

Le tunnel du chemin de fer de Vierzy s'est effondré en juin 1972 pendant des travaux d'entretien après une très longue période de service marquée par des réparations liées à l'ancienneté et à des destructions militaires.

Mots-clés : tunnel de chemin de fer, effondrement.

\section{The collapse of the Vierzy railway tunnel}

After a very long time since the tunnelling of the Vierzy railway tunnel it collapsed all of a sudden.

Key words: railway tunnel, collapse, 
A l'encontre des cas cités au cours cette réunion, l'effondrement du tunnel de Vierzy le 16 juin 1972 n'a pas apporté grand-chose à la mécanique des roches, si ce n'est de confirmer que la rupture des roches peut se produire après de très long temps, comme cela a eu lieu dans certains stots des mines de fer de Lorraine ou lors de l'effondrement de Clamart. Cependant, comme cette catastrophe a fait une centaine de morts on ne peut pas négliger d'en parler.

Le tunnel de Vierzy a une longueur de $1394 \mathrm{~m}$; il avait été construit dix ans avant la guerre de 1870 , sous un recouvrement de $40 \mathrm{~m}$. Sa réalisation avait été faite dans des conditions tout à fait favorables, dans les sables de Cuise directement sous les calcaires grossiers de la région parisienne. On ne pouvait pas rêver mieux. Il n'y avait pas d'eau ou très peu, et localement ; le toit tenait plutôt bien; il était soutenu par des parements en bonne maçonnerie de blocs de calcaire grossier prélevés et taillés en carrière, mais probablement sans attendre la dessiccation des pierres à l'air, ce qui, comme on le sait, renforce la résistance des calcaires, même si la pierre est ensuite ré-humidifiée. Évidemment les propriétés du toit calcaire pouvaient varier d'un point à un autre car la base des Calcaires Grossiers c'est le Lutétien qui n'est pas aussi bon que le Banc royal...

Malheureusement, le site de ce tunnel était vrai ment très mal placé : au cours des guerres de 1870 , de 1914-1918, de 1939-1945, toutes les armées en retraite qui passaient par là ont saboté le tunnel en partant, de façon à entraver la marche de leurs adversaires. Au moins quatre fois; ce qui évidemment n'a pas été très bon pour la santé de l'ouvrage...

Vers 1930 , certains parements avaient montré en hiver des signes de fatigue, avec des dégradations en surface, voire de l'écaillage de la maçonnerie, et à partir de cette alerte on a diagnostiqué une gélivité de la pierre. Cette hypothèse était vraisemblable car la région de Villers-Cotterêts jusqu'à Soissons et même vers Reims peut être très froide en hiver, avec des températures jusqu'à $-15^{\circ} \mathrm{C}$. Il fut donc décidé de remplacer le soutènement par des panneaux préfabriqués de briques. La situation ainsi améliorée a été surveillée et a montré à nouveau, après quelques dizaines d'années, des dégradations superficielles des briques que l'on a alors sans doute considérées elles aussi comme probablement gélives. La SNCF décida alors un traitement plus lourd sur $71 \mathrm{~m}$ de longueur avec un décaissage des revêtements par courtes longueurs, la mise en place d'un treillis soudé puis d'un béton projeté de sept centimètres d'épaisseur, les creux de la voûte après clémolition du mortier de liaison ayant été garnis de béton venant en surépaisseur : cette remise en état était donc beaucoup plus importante et plus efficace que l'intervention précédente.

Alors là, trois malchances se produisent. D'abord le compresseur, nécessaire pour la projection du béton, tombe en panne le 5 avril. Pour ne pas retarder les travaux, il est alors décidé de les reprendre par le nord, c'est-à-dire par l'autre bout de la rénovation de $71 \mathrm{~m}$ toujours par longueurs courtes de $7 \mathrm{~m}$ et en revenant vers ce qui avait déjà été fait, c'est-à-dire les $6 \mathrm{~m}$ déjà découverts. Les travaux sont presque terminés et le soir du dernier jour, les derniers $6 \mathrm{~m}$ ayant été décaissés, la journée de travail étant finie sans problème apparent, tout le monde quitte le chantier et le tunnel. Ultérieurement, une locomotive isolée passe dans le tunnel. Ultérieurement encore, après un délai non identifié, le tunnel s'effondre et c'est là la deuxième malchance : car si le travail n'avait pas été abandonné il est probable que les travailleurs se seraient rendu compte de l'imminence de l'effondrement; ils auraient alors pu prendre, peut-être, quelques précautions, mais surtout faire prévenir les trains que la voie n'était plus libre. De plus si les ouvriers en partant n'avaient pas encore quitté le tunnel, ils auraient probablement entendu le vacarme de l'effondrement. Et que dire du passage de la locomotive isolée ? A partir de ce moment, il n'y avait plus personne dans le tunnel, mais un énorme tas de pierres au milieu des voies et au-dessus une grande cloche d'effondrement à la place du toit. La longueur de l'éboulement était de 14 mètres.

Puis vient l'autorail de Paris vers Soissons, à $108 \mathrm{~km} / \mathrm{h}$; à 20 h 55 la motrice monte sur le tas de pierres et va s'encastrer dans la cloche du toit.

Puis vient l'autorail de Soissons vers Paris, à $90 \mathrm{~km} / \mathrm{h}$, qui s'écrase à son tour à 20 h 56 sur le tas de pierres. Dans les deux cas, aucune trace de freinage n'a été trouvée.

On imagine alors l'horreur du drame, dans l'obscurité, avec les cris des blessés sans secours, ou des secours qui ne pourront parvenir qu'au bout d'un temps très long...

En fait, une dalle de calcaire du Lutétien à l'emplacement de l'effondrement s'était détachée de la base des calcaires grossiers et s'appuyait sur le tunnel. Celui-ci avait commencé à (c souffrir ) d'une très lente rupture différée qui s'était manifestée par des dégradations sur des parements, qu'ils soient en pierres ou en briques. Et, voilà la troisième malchance : c'est le fait que la panne de compresseur se soit produite justement en ce lieu très particulier où une dalle calcaire s'était décollée, où un joint de stratification s'était ouvert au-dessus d'elle déjà depuis un temps très long et où cette dalle surchargeait le tunnel, d'où les désordres observés. Mais depuis quand? Certainement depuis très longtemps, car dans le vide du décollement on a trouvé les traces noirâtres de la fumée des convois, c'est-à-dire des traces très anciennes, et d'ailleurs sans qu'on sache très bien par quelles fissures ces fumées avaient pu circuler dans ce joint. Les vibrations engendrées par le passage des trains ont donc eu le temps, bien avant la catastrophe, de secouer cet emplacement désormais très sensible.

La figure 1 représente le profil en long de la partie effondrée du tunnel de Vierzy avec le massif sur lequel les convois se sont brisés.

La figure 2 est une photographie de la brèche d'effondrement du côté de Soissons. On y voit nettement le banc de calcaire qui s'est plié pour "( s'asseoir »sur la voûte du tunnel.

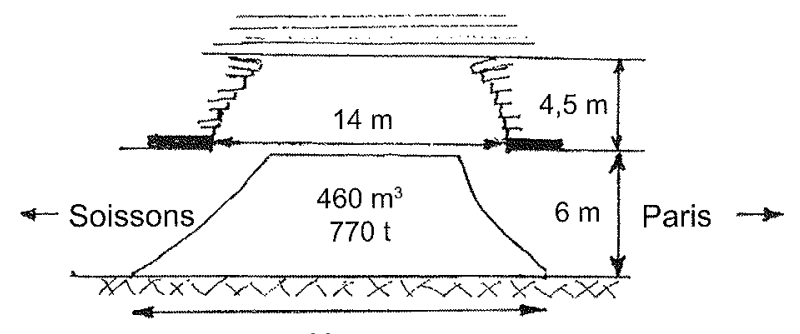

$20 \mathrm{~m}$

Fo. 1 Profil en long de l'effondrement du tunnel de Vierzy. 


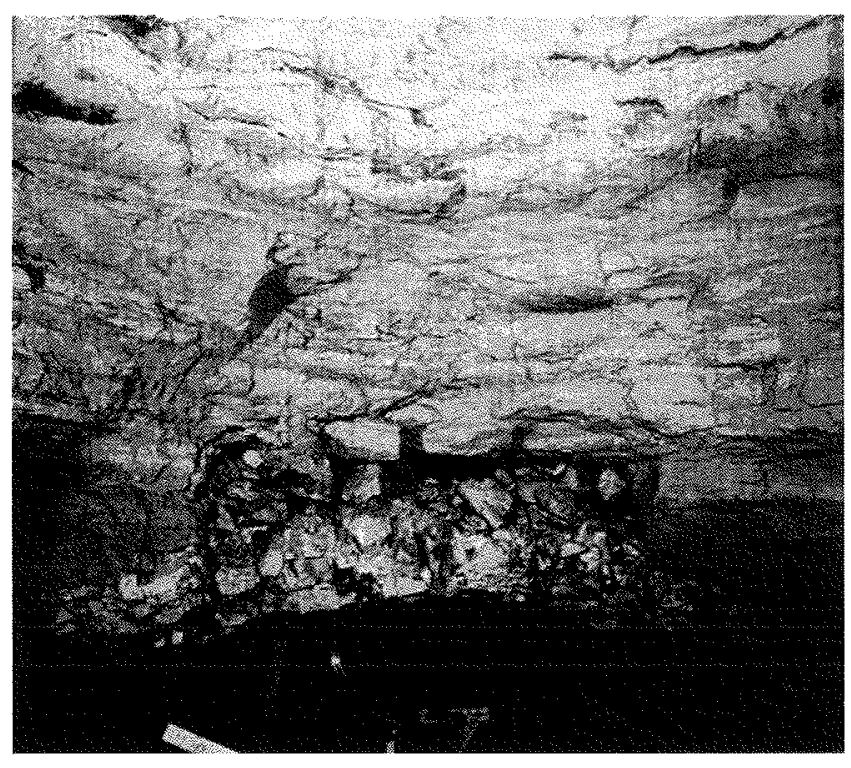

न. 2 Effondrement du tunnel côté Soissons

La figure 3 est une photographie à $14 \mathrm{~m}$ au sud de la précédente qui représente la brèche d'effondrement du côté de Vierzy. On y voit distinctement le panneau de briques de la précédente réparation qui n’a pas encore été enlevé, ainsi que la galerie pilote au toit du tunnel, plus ou moins bien tassée et liée au Lutétien et surtout tout en haut, au plafond, la fissure au-dessus du banc décollé de la masse effondrée.

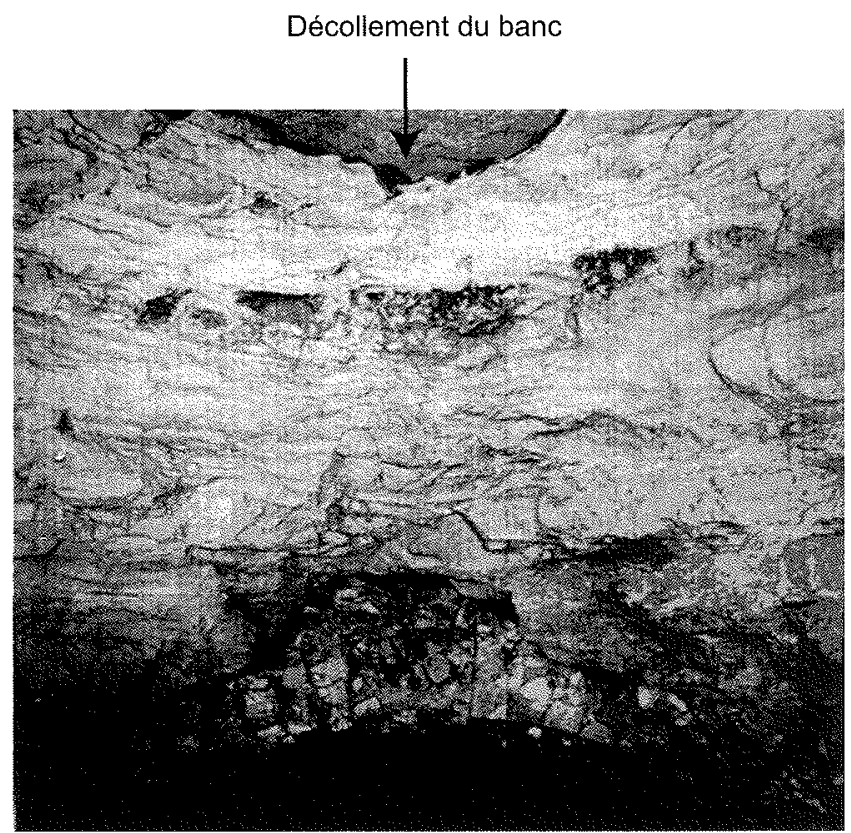

16. 3 . Effondrement du tunnel côté Vierzy.
L'effondrement du tunnel de Vierzy a mis beaucoup de temps à se produire entre le moment d'un chargement excessif et la rupture franche. Mais quand a lieu le chargement excessif ? Probablement au cours d'un sabotage du tunnel. Mais quel sabotage puisqu'il y en a eu plusieurs ! L'écaillage observé en parement a été attribué à la gélivité des pierres calcaires, ce qui est à peine crédible car le tunnel, dès la construction, est apparu comme étant pratiquement sec, sauf localement. Quant à l'écaillage des rouleaux de briques, il peut difficilement être justifié par la gélivité des briques et apparaît davantage devoir être attribué à une contrainte tangentielle presque excessive qui s'achemine vers une rupture différée. Ce cheminement est un des sujets les plus difficiles de la Mécanique des Roches. L'effondrement différé de Vierzy peut être rapproché de celui de la carrière de craie de Clamart, Issy-les-Moulineaux, par la durée de son apparente stabilité

Vers les années 30, Armand Mayer (qui fut bien plus tard à l'origine de la création du Comité français de mécanique des roches), ingénieur des Mines à l'Inspection générale des carrières de Paris, fut chargé d'expertiser cette carrière qui paraissait un peu douteuse. Il fit réaliser des essais de compression simple sur des éprouvettes de craie prélevées dans la carrière pour les comparer à la contrainte dans les piliers, calculée à partir du poids des terres et de la surface des sections droites des piliers de craie. En somme une mécanique des roches élémentaire. La résistance de la craie étant le double de la contrainte moyenne dans les piliers il trouva que ce coefficient de sécurité était trop petit et le terrain de surface fut déclaré inconstructible.

Après la guerre de 1939-1945, rien ne s'étant produit depuis vingt ans, la poussée démographique aidant, le terrain fut utilisé pour des constructions relativement légères par rapport au poids des terres. En 1961, l'effondrement de six hectares engendra de gros dégâts en surface avec des immeubles détruits, une vingtaine de décès et de nombreux blessés. Armand Mayer avait eu tort pendant trente ans. Mais il a eu raison après.

Le cas des falaises qui s'effondrent après de nombreuses années est moins significatif car les tempêtes, la température, la météo peuvent jouer. Mais le calme d'une carrière fermée est bien plus difficile à commenter et montre la difficulté de la prévision de la rupture différée.

\section{$\overline{\text { Bibliographie }}$}

Rapport de l'enquète administrative du 11 mars 1973, présidée par J. Rérolle (J. Goguel, J. Kérisel, F. Ramel, L. Lupiac et P. Duffaut). Publié au J.O. du 11 avril 1973, p. 53 à 72. 